\title{
MRI Findings in Patients with a History of Failed Prior Microvascular Decompression for Hemifacial Spasm: How to Image and Where to Look
}

\author{
M.A. Hughes, B.F. Branstetter, C.T. Taylor, S. Fakhran, W.T. Delfyett, A.M. Frederickson, and R.F. Sekula Jr
}

\begin{abstract}
BACKGROUND AND PURPOSE: A minority of patients who undergo microvascular decompression for hemifacial spasm do not improve after the first operation. We sought to determine the most common locations of unaddressed neurovascular contact in patients with persistent or recurrent hemifacial spasm despite prior microvascular decompression.
\end{abstract}

MATERIALS AND METHODS: Eighteen patients with a history of a microvascular decompression presented with persistent hemifacial spasm. All patients underwent thin-section steady-state free precession MR imaging. Fourteen patients underwent repeat microvascular decompression at our institution. Images were evaluated for the following: the presence of persistent vascular compression of the facial nerve, type of culprit vessel (artery or vein), name of the culprit artery, segment of the nerve in contact with the vessel, and location of the point of contact relative to the existing surgical pledget. The imaging findings were compared with the operative findings.

RESULTS: In 12 of the 18 patients (67\%), persistent vascular compression was identified by imaging. In 11 of these 12 patients, the culprit vessel was an artery. Compression of the attached segment (along the ventral surface of the pons) was identified in most patients ( $58 \%$, $7 / 12$ ). The point of contact was proximal to the surgical pledget in most patients $(83 \%, 10 / 12)$. The imaging interpretation was concordant with the surgical results regarding artery versus vein in $86 \%$ of cases and regarding the segment of the nerve contacted in $92 \%$.

CONCLUSIONS: In patients with persistent hemifacial spasm despite microvascular decompression, the unaddressed vascular compression is typically proximal to the previously placed pledget, usually along the attached segment of the nerve. Re-imaging with highresolution T2-weighted MR imaging will usually identify the culprit vessel.

ABBREVIATIONS: AICA = anterior inferior cerebellar artery; HFS = hemifacial spasm; MVD = microvascular decompression; PICA = posterior inferior cerebellar artery; SSFP = steady-state free precession

$\mathrm{H}$

emifacial spasm (HFS) is characterized by unilateral spasms of the facial musculature. ${ }^{1}$ While not life-threatening, the disease can profoundly reduce quality of life. It has an annual incidence of approximately 1 in 100,000 people per year. ${ }^{2}$ HFS is most commonly the result of vascular compression of the facial nerve. ${ }^{3}$ Microvascular decompression (MVD) of the facial nerve is a well-established treatment for HFS with success rates exceed-

Received August 5, 2014; accepted after revision September 30.

From the Departments of Radiology (M.A.H., B.F.B., C.T.T., S.F., W.T.D.), Neurosurgery (A.M.F., R.F.S.), and Otolaryngology (B.F.B.), University of Pittsburgh Medical Center, Pittsburgh, Pennsylvania.

Paper previously presented at: American Society of Neuroradiology Annual Meeting and the Foundation of the ASNR Symposium, May 17-22, 2014; Montreal, Quebec, Canada.

Please address correspondence to Raymond F. Sekula Jr, MD, University of Pittsburgh Medical Center, Department of Neurological Surgery, 200 Lothrop St, Suite B-400, Pittsburgh, PA 15213; e-mail: sekularf@upmc.edu

http://dx.doi.org/10.3174/ajnr.A4174 ing $90 \%$ for the initial operation. ${ }^{4}$ Patients who have persistent HFS despite undergoing MVD pose a challenge for both neuroradiologists and neurosurgeons. However, many patients with unabated HFS despite prior MVD can and do benefit from repeat operations. ${ }^{5,6}$ Failure to identify persistent vascular compression of the facial nerve can discourage reoperation and potential cure. The purpose of this article was to determine whether MR imaging could identify unaddressed neurovascular contact in patients with ongoing HFS despite prior MVD and to report the frequency and most common locations of this residual neurovascular contact.

\section{MATERIALS AND METHODS Study Population}

This retrospective review was approved by the institutional review board at the University of Pittsburgh Medical Center and was Health Insurance Portability and Accountability Act-compliant. 
MVD imaging protocol ${ }^{\mathrm{a}}$

\begin{tabular}{lccccccc}
\hline $\begin{array}{c}\text { Sequence and } \\
\text { Plane }\end{array}$ & $\begin{array}{c}\text { Flip } \\
\text { Angle }\end{array}$ & $\begin{array}{c}\text { FOV } \\
(\mathbf{c m})\end{array}$ & TR/TE $(\mathbf{m s})$ & $\begin{array}{c}\text { Section } \\
\text { Thickness } \\
\text { (mm) }\end{array}$ & $\begin{array}{c}\text { Spacing } \\
\text { (mm) }\end{array}$ & Matrix \\
\hline SSFP-axial & $65^{\circ}$ & 18 & Default to minimum & 1 & 0.5 & $384 \times 256$ \\
SSFP-coronal & $65^{\circ}$ & 18 & Default to minimum & 1 & 0.5 & $384 \times 256$ \\
SSFP-sagittal & $65^{\circ}$ & 20 & Default to minimum & 1 & 0.5 & $384 \times 256$ \\
\hline
\end{tabular}

${ }^{a}$ Also includes routine whole-brain sagittal T1-weighted, axial FLAIR, and diffusion sequences.

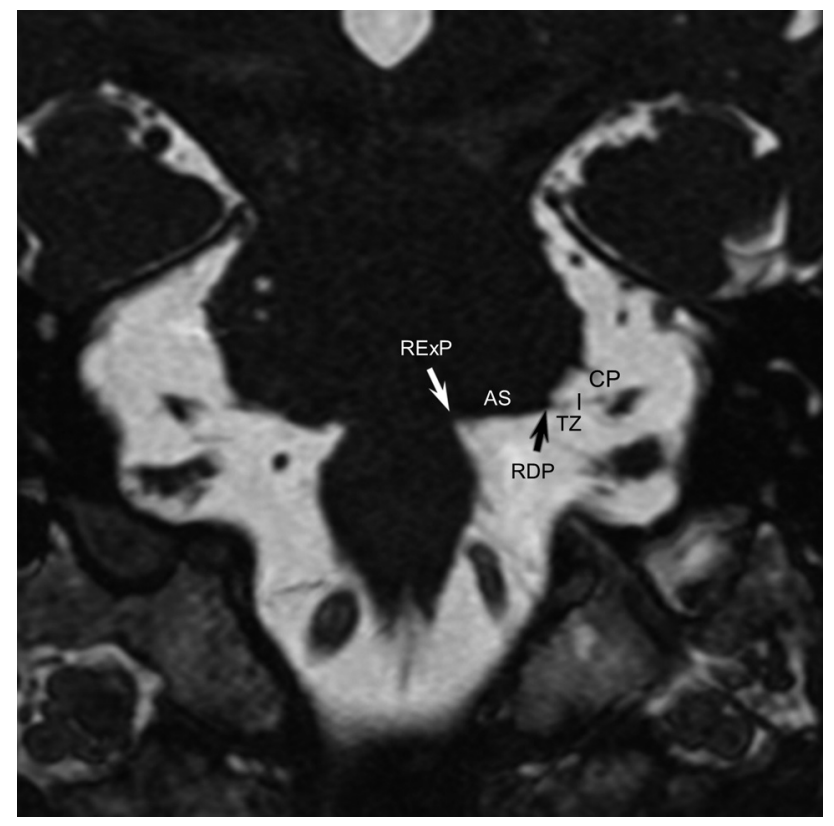

FIG 1. Facial nerve anatomy. Coronal SSFP image shows the expected locations of the root exit point (REXP, white arrow), the attached segment (AS) along the undersurface of the pons, and the root detachment point (RDP, black arrow). The transition zone (TZ) extends for approximately 3-4 $\mathrm{mm}$ anterolateral to the root detachment point. The cisternal portion of the facial nerve $(\mathrm{CP})$ extends laterally toward the porous acusticus.

Patients presenting with persistent HFS despite prior MVD between January 2011 and December 2013 to a single neurosurgeon specializing in cranial nerve disorders were included in this study. Inclusion criteria were the following: history of a prior MVD, persistent HFS, availability of a thin-section steady-state free precession (SSFP) MR imaging performed after the date of the unsuccessful prior MVD, and having undergone a repeat MVD at our institution.

\section{MR Imaging Technique}

Patients with HFS who are being considered for repeat MVD undergo MR imaging at our institution tailored to delineate the facial nerve and adjacent vessels. ${ }^{7}$ Studies are performed on either 1.5T or 3T MR imaging scanners (Optima and Discovery; GE Healthcare, Milwaukee, Wisconsin) and include whole-brain sagittal T1, axial FLAIR, and DWI sequences. Thin-section axial, coronal, and sagittal SSFP images through the brain stem are obtained. The imaging protocol is summarized in the Table.

\section{Anatomic Terms}

We used anatomic terms initially proposed by Tomii et $\mathrm{al}^{8}$ in 2003 and expanded by Campos-Benitez and Kaufmann. ${ }^{9}$ The facial nerve emerges from the brain stem within the pontomedullary sulcus at the root exit point. The facial nerve then adheres to the ventral surface of the pons for $8-10 \mathrm{~mm}$, which is called the attached segment. It then separates from the pons at the root detachment point. The next segment is the transition zone of the facial nerve, which is an approximately 4-mm segment of the facial nerve where the central glial myelin transitions into the peripheral myelin created by Schwann cells. The central glial myelin does not exceed a point 4-mm distal to the root detachment point of the facial nerve. ${ }^{8}$ After the transition zone, the cisternal portion of the nerve extends anterolaterally to the porous acusticus. The component of the nerve within the internal auditory canal is the canalicular segment. These anatomic/histologic segments can be approximated on thin-section SSFP MR imaging (Fig 1). The relevant facial nerve segments are also depicted in Fig 2.

\section{Neurosurgical Clinical Evaluation}

All patients were evaluated by a neurosurgeon (R.F.S.) who has 9 years of experience specializing in cranial nerve disorders and has performed >500 MVD operations for HFS. The diagnosis of persistent HFS was based on history and clinical evidence of persistent HFS on examination and was supported by electromyography.

\section{Image Interpretation}

Blinded to the surgical results, a single Certificate of Added Qualification-certified neuroradiologist (M.A.H.) reviewed the imaging to identify the following: the presence of a vessel contacting the facial nerve; whether the contacting vessel was an artery, vein, or both; which artery was responsible; which segment of the nerve was contacted; and whether this point of contact was proximal or distal to the existing pledget. A single neurosurgeon (R.F.S.) performed all repeat MVDs, and the imaging results were compared with his operative notes and detailed drawings performed after each case. Descriptive statistics were tabulated.

\section{Surgical Technique}

MVD was performed in the manner previously described. ${ }^{10,11}$ In brief, the cerebellopontine angle was accessed through a retromastoid craniectomy measuring approximately $2 \times 1.5 \mathrm{~cm}$ without the use of fixed retraction. The centrally myelinated portion of the facial nerve, from the root exit point to $4 \mathrm{~mm}$ distal to the root detachment point, was inspected for residual compression. Monopolar stimulation was used to assist in identifying the nerve along the attached segment. Any vascular compression found along this portion of the facial nerve was decompressed by using shredded Teflon (Dupont, Wilmington, Delaware) pledgets of varying sizes. On occasion, the previously achieved decompression was bolstered with additional pledgets. Of note, the presence of scarring and adhesions typically makes operative exposure of the facial nerve more difficult in repeat MVD operations, sometimes adding an additional hour to the operative time. 


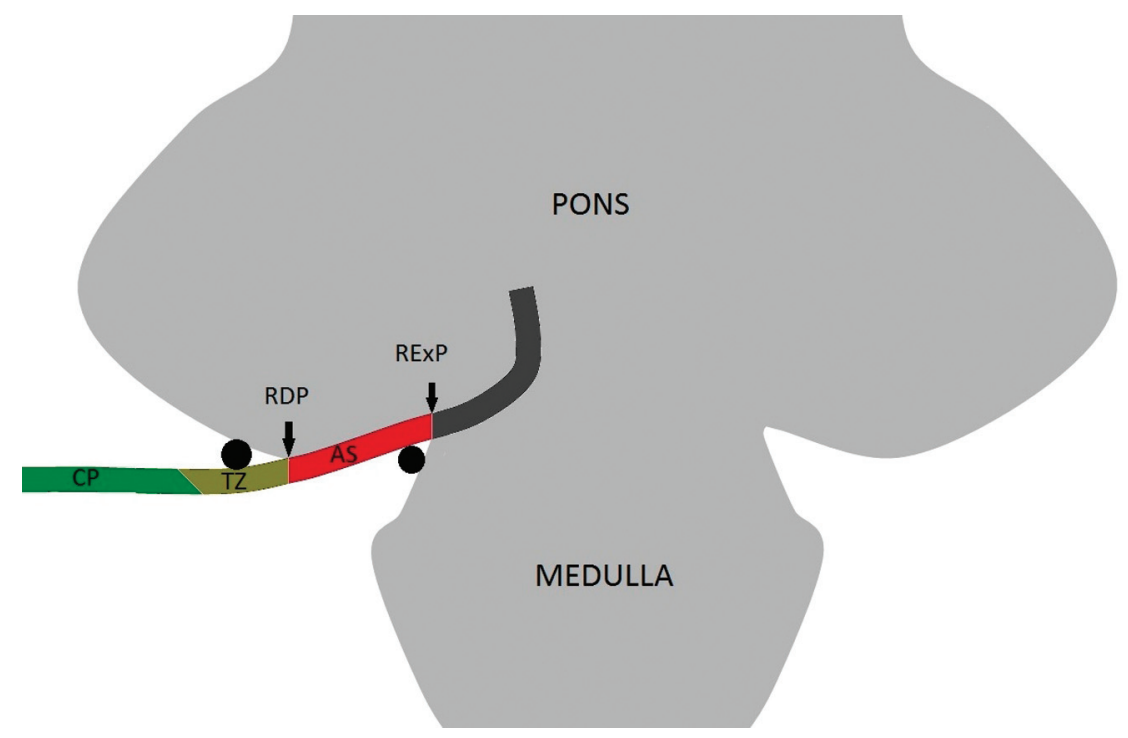

FIG 2. Coronal drawing demonstrates the facial nerve exiting the brain stem at the root exit point (RExP) in the pontomedullary sulcus. The attached segment (AS) of the nerve then runs along the pons until the nerve separates from the brain stem at the root detachment point (RDP). The transition zone (TZ) is the 3- to 4-mm segment of the facial nerve where the central glial myelin transitions into the peripheral myelin. The cisternal portion (CP) of the facial nerve is noted laterally. Black circles demonstrate common points of arterial compression of the facial nerve.
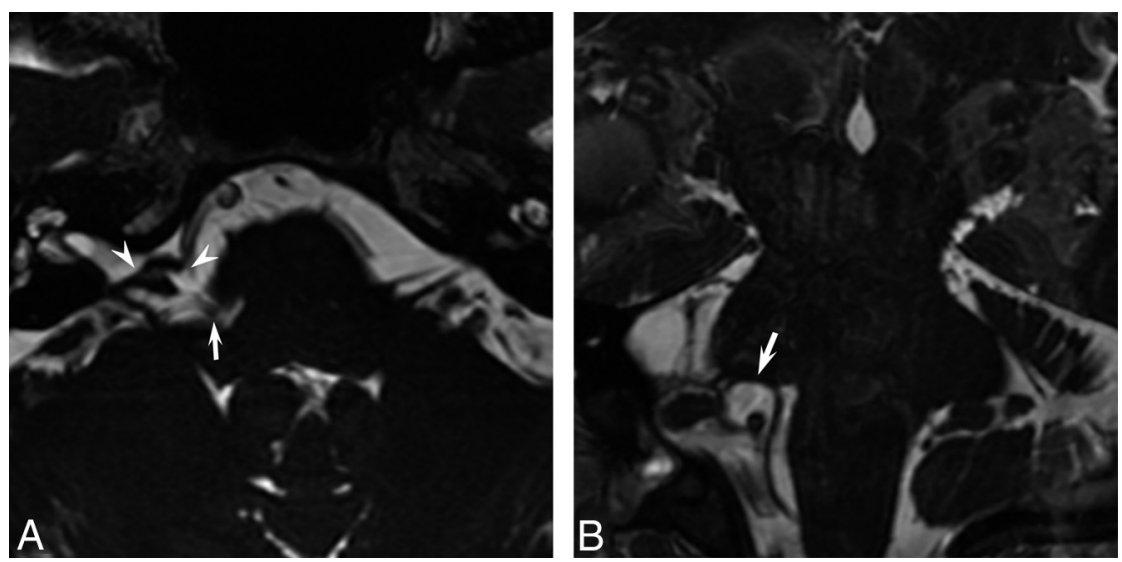

FIG 3. A, Axial SSFP image demonstrates the low-signal Teflon pledget (white arrowheads) in the right cerebellopontine angle, indicating a prior surgical decompression of the cisternal portion of the facial nerve. The right PICA is located medial to the pledget in the pontomedullary sulcus (white arrow). B, On a coronal SSFP image, the right PICA is noted to contact and deform the undersurface of the pons along the attached segment of the facial nerve (white arrow). This culprit vessel was confirmed and treated surgically, with complete resolution of facial spasms.

\section{Clinical Follow-Up}

Patients were contacted postoperatively via phone by a disinterested observer (A.M.F.). He administered a detailed questionnaire that addressed the presence or absence of HFS. Patients were asked to characterize their outcome after each procedure as complete spasm relief, $>75 \%$ spasm relief, $>50 \%$ spasm relief, or $<50 \%$ spasm relief. To better assess quality of life following repeat MVD for HFS, patients were asked, "Would you elect to undergo the same procedure again if you found yourself in similar circumstances?"12

\section{RESULTS}

Eighteen patients with a history of a prior MVD presented with persistent HFS. Fourteen patients were offered and subsequently underwent a repeat MVD at our institution. Twelve of the 14 patients had their initial operation at an outside institution. The age range of patients at the time of the operation was 27-65 years; mean, 49 years. Ten patients were women and 4 were men. The persistent HFS was right-sided in 9 patients and left-sided in 5 patients.

Of the 14 patients included in our study, 12 (86\%) had imaging evidence of persistent vascular compression of the facial nerve. The posterior inferior cerebellar artery (PICA) was the sole culprit vessel in 5 patients $(42 \%, 5 / 12)$, the anterior inferior cerebellar artery (AICA) was the sole culprit vessel in 5 patients $(42 \%, 5 / 12)$, and both the AICA and PICA contacted the facial nerve in 1 patient $(8 \%, 1 / 12)$. A vein was identified contacting the facial nerve in 1 patient $(8 \%, 1 / 12)$. No neurovascular contact was seen on MR imaging in 2 patients. Of the patients with vascular contact, the location was the following: the attached segment in 7 patients $(58 \%$, $7 / 12)$, the root detachment point in 1 patient $(8 \%, 1 / 12)$, and the transition zone in 4 patients $(33 \%, 4 / 12)$. The point of contact was proximal to the pledget in $10 \mathrm{pa}$ tients $(83 \%, 10 / 12)$ and distal to the pledget in 2 patients $(17 \%, 2 / 12)$. Thus, persistent neurovascular contact was along the more proximal portion of the facial nerve in most patients. Imaging examples demonstrating proximal unaddressed vascular compression of the facial nerve following the first MVD are presented in Figs 3 and 4.

Three patients without imaging evidence of arterial compression on retrospective imaging review underwent repeat surgical decompression. Intraoperatively, only small arterioles were noted contacting the attached segment of the facial nerve in 2 of these patients (Fig 5). In the third patient, the AICA was identified contacting the attached segment intraoperatively, but only a vein was identified contacting the facial nerve on imaging.

Four patients were not offered repeat decompression. Three of these patients had no evidence of persistent vascular compression, and 1 patient had arterial contact of the cisternal portion (peripherally myelinated segment) of the facial nerve, distal to the existing pledget.

The imaging interpretation was concordant with the surgical results regarding artery versus vein in 12 patients $(86 \%, 12 / 14)$. In 1 patient, a vein contacting the transition zone was identified by imaging, but intraoperatively, the AICA was noted to contact the attached segment. In the other patient, the AICA was noted con- 

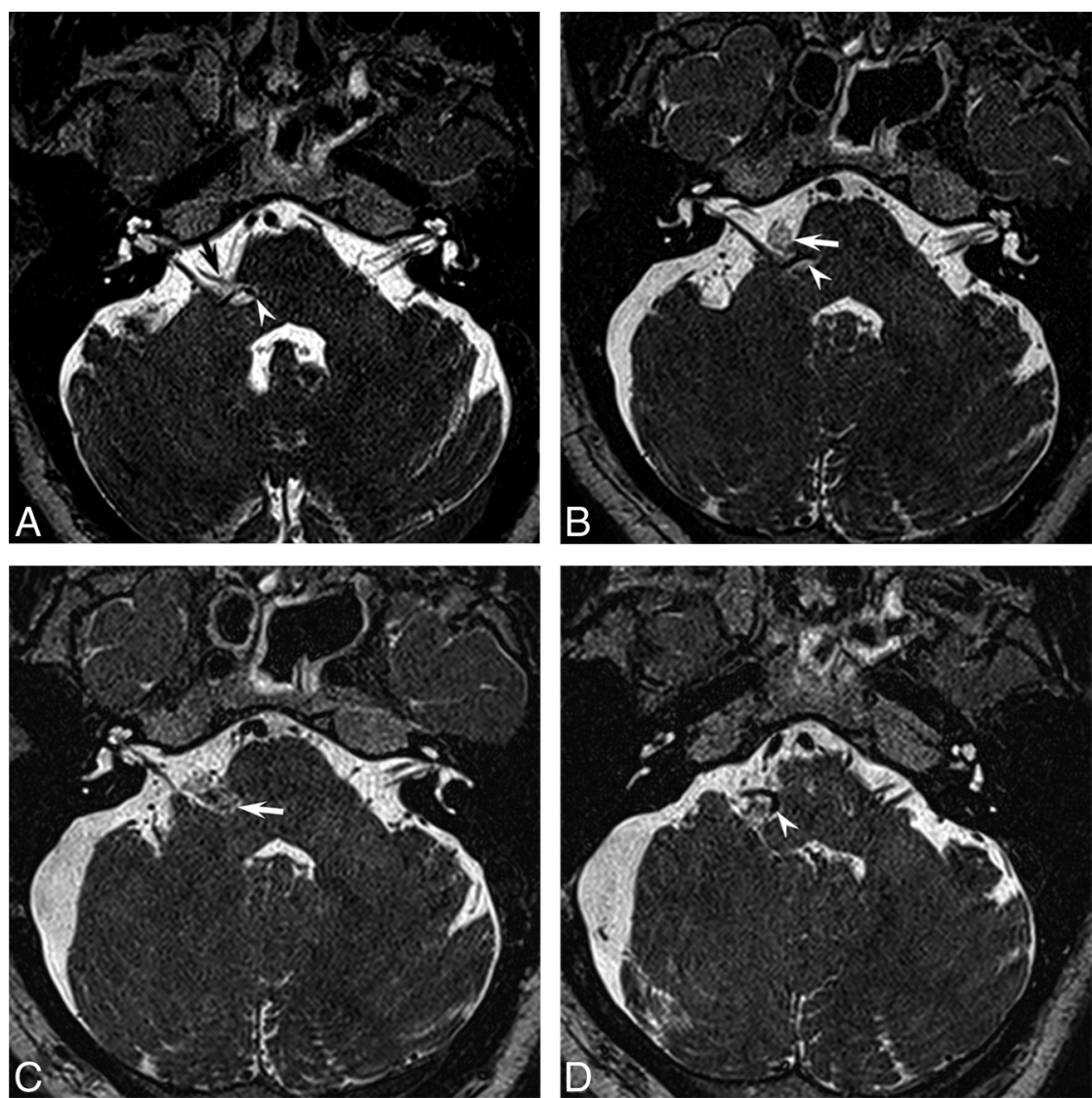

FIG 4. A, Preoperative axial image demonstrates 2 points of arterial contact of the facial nerve: The right AICA contacts the transition zone of the facial nerve (black arrow) and the right PICA contacts the attached segment of the nerve (white arrowhead). B. Postoperative axial image following the initial MVD demonstrates a Teflon pledget (white arrow) lateral to the pons, which decompressed the AICA from the transition zone of the facial nerve. Note that the PICA along the attached segment (white arrowhead) remains unaddressed. The patient had no significant reduction in spasms following this operation. $C$ and $D$, Postoperative axial images following the second MVD demonstrate an additional pledget (white arrow, $C$ ) decompressing the attached segment of the nerve and displacing the PICA inferiorly (white arrowhead, D). The patient was spasm-free following the second MVD.

tacting the transition zone but intraoperatively only venous contact of the facial nerve was noted. In the 11 patients with arterial contact of the facial nerve identified by imaging who underwent repeat MVD, the radiologist's naming of the vessel was concordant with the surgeon's findings in 7 patients $(63 \% 7 / 11)$. In the 12 patients with neurovascular contact identified by imaging (either arterial or venous), the imaging findings regarding the segment of the nerve contacted were concordant with the surgical findings in 11 patients $(92 \%, 11 / 12)$.

Of the 14 patients who underwent repeat MVD, 12 were successfully contacted via phone and questioned regarding continued symptoms. The follow-up range was 4.0-35.9 months following the repeated MVD, with a median follow-up duration of 12.7 months. Nine of 12 patients were either spasm-free (7 patients) or had $>75 \%$ reduction in their spasm frequency ( 2 patients). Two patients had no improvement in the frequency of their spasms, and 1 patient reported a $<50 \%$ reduction, specifically a $20 \%$ reduction in spasms. Ten patients $(83 \%, 10 / 12)$ contacted said that they would repeat the operation if they found themselves in similar circumstances. Of the 2 patients with no improvement in spasms, 1 had arterial contact along the most distal aspect of the transition zone where the centrally myelinated portion of the nerve transitions into the more resistant peripherally myelinated portion, and the other patient had only venous contact of the facial nerve identified by imaging and was also 1 of the 2 patients in whom the imaging and surgical results regarding artery versus vein were discordant.

\section{DISCUSSION}

MVD is the only permanent treatment of HFS and has a high success rate of approximately $90 \%$ for the initial surgery in experienced hands. ${ }^{5,13-15}$ Reasons for an unsuccessful MVD include not identifying the true culprit vessels and incomplete vascular decompression. ${ }^{16}$ Additionally, a subpopulation of $<10 \%$ of patients do not improve despite undergoing a technically successfully procedure, possibly due to a central mechanism., ${ }^{5,13-16}$ The existing literature regarding surgical success rates for repeat MVD in the setting of persistent HFS is less extensive. The anatomic location along the facial nerve where persistent vascular compression was identified in repeat MVD surgeries has not been well-described in the existing literature. Engh et $\mathrm{al}^{6}$ performed 41 repeat MVDs for HFS and reported a favorable outcome in $82.4 \%$ of patients; however, the sites of persistent compression of the facial nerve relative to the prior surgical decompression site were described in only 2 cases. Zhong et $\mathrm{al}^{13}$ reported results on 30 patients who underwent repeat MVD for persistent HFS, with all patients demonstrating improvement postoperatively. The authors reported that the failed first MVD was because the true culprit vessel was more proximal than expected. The authors further described the culprit artery as rostral to the facial nerve in 9 patients, between the seventh and eighth nerve in 9 patients, along the cisternal portion in 7 patients, and in the pontomedullary fissure in 15 patients. Wang et $\mathrm{al}^{16}$ reported the surgical outcome of 33 patients who underwent repeat MVD, with $85 \%$ of patients experiencing long-term relief, but they did not delineate the sites of persistent compression.

Unknown by many physicians and of critical importance is that the location of neurovascular contact resulting in HFS is often along the attached segment of the facial nerve along the ventral surface of the pons. In a study of 115 patients undergoing their first MVD for HFS, the contact location in most patients (64\%) was the attached segment. ${ }^{9}$ In our study, persistent neurovascular contact along the attached segment was identified by imaging in $50 \%(7 / 14)$ of all patients and in 58\% (7/12) of patients with radiologically identifiable culprit vessels. Thus, the attached seg- 


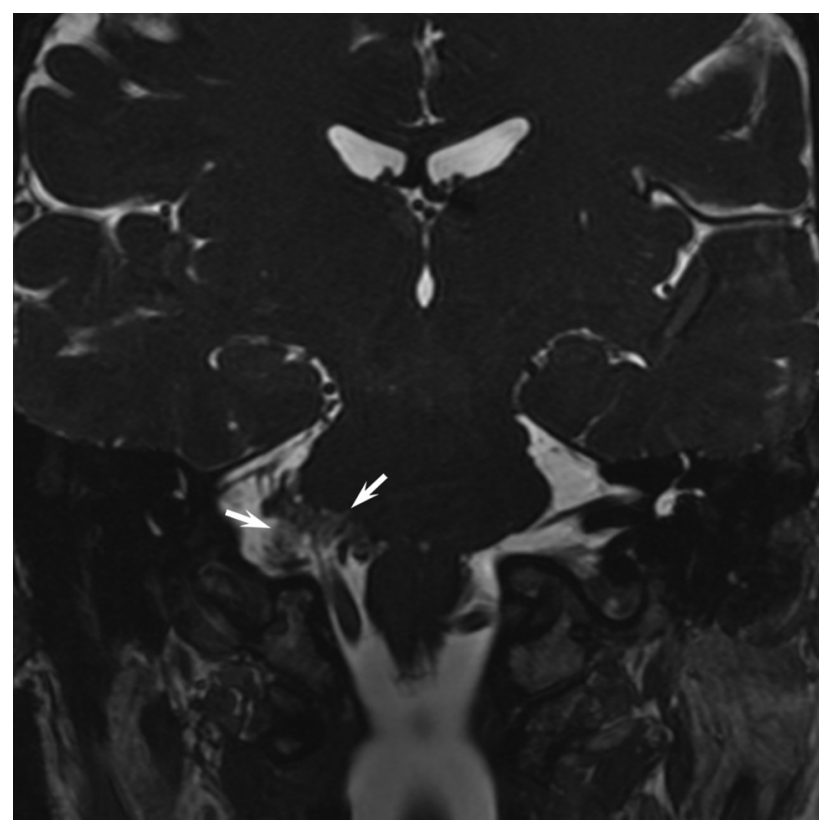

FIG 5. Coronal SSFP image demonstrates a large pledget (white arrow) along the attached segment of the facial nerve. No residual vascular compression of the nerve was identified by imaging. Intraoperatively, the previously placed pledget was removed and a small arteriole was identified contacting the attached segment of the nerve. The arteriole was addressed, and the patient was spasm-free postoperatively. This case illustrates how difficult it can be to interpret these imaging studies in the setting of prior pledget placement.

ment of the facial nerve extending along the ventral surface of the pons must be carefully scrutinized in all patients with HFS, including those who have undergone prior MVD. With improved MR imaging techniques, there is increasing reliance on imaging to identify vascular compression before both the first MVD and on any subsequent repeat operations. If a radiologist does not identify a site of persistent compression when present and instead reports that the facial nerve is well-decompressed, a patient may not be offered surgery and thus be denied a potential cure.

Historically, neurosurgeons have used MR imaging in the evaluation of patients with HFS to rule out confounding diagnoses such as neoplasms, vascular malformations, and multiple sclerosis. ${ }^{14}$ However, improvements in MR imaging have led to an increased interest in its use for evaluating vascular compression of the cranial nerves. ${ }^{17-20}$ The use of SSFP imaging has been shown to predict vascular compression of the facial nerve with sensitivities of $75 \%-93 \%$ and specificities of $29 \%-75 \%$ for blinded observers. ${ }^{21}$ In light of this higher sensitivity and lower specificity, the decision to operate is supported by imaging, but imaging results are never the sole factor in determining whether to offer MVD to a patient. Specifically, at our institution, an operation is typically not offered to a clinically unfavorable candidate even if imaging demonstrates vascular compression of the facial nerve. However, a clinically favorable candidate will be warned that MVD has a lower chance of success if imaging does not clearly demonstrate a compressive vessel.

This study was limited by being a single-center retrospective study with a small number of patients. HFS is a rare disease that has a high surgical cure rate with initial surgeries, so the potential number of patients with persistent HFS following MVD is low. A single neuroradiologist reviewed the images retrospectively; the original reports of the studies were not used because as neuroradiologists, our understanding of the proximal facial nerve anatomy and in particular the more susceptible centrally myelinated portions of the nerve has increased rapidly during the past 2 years after a neurosurgeon specializing in cranial nerve disorders joined our institution. Many of the initial reports described vascular contact of the facial nerve only along its cisternal portion. Finally, preoperative SSFP imaging before the initial MVD was not available in most of our patients $(86 \%, 12 / 14)$. This fact limits our ability to definitively confirm the original point of neurovascular contact.

\section{CONCLUSIONS}

Patients with persistent HFS despite prior MVD often have identifiable persistent neurovascular compression along the centrally myelinated susceptible portion of the facial nerve when imaged with dedicated thin-section SSFP sequences. In most patients, this compression occurs proximal to the existing surgical pledget. The attached segment of the facial nerve must be closely scrutinized to identify persistent neurovascular contact and thus guide the surgical management of these patients.

\section{REFERENCES}

1. Tan NC, Chan LL, Tan EK. Hemifacial spasm and involuntary facial movements. QJM 2002;95:493-500

2. Auger RG, Whisnant JP. Hemifacial spasm in Rochester and Olmsted County, Minnesota, 1960 to 1984. Arch Neurol 1990;47:1233-34

3. Jannetta PJ, Abbasy M, Maroon JC, et al. Etiology and definitive microsurgical treatment of hemifacial spasm: operative techniques and results in 47 patients. J Neurosurg 1977;47:321-28

4. Miller LE, Miller VM. Safety and effectiveness of microvascular decompression for treatment of hemifacial spasm: a systematic review. Br J Neurosurg 2012;26:438-44

5. Sekula RF Jr, Frederickson AM, Arnone GD, et al. Microvascular decompression for hemifacial spasm in patients $>65$ years of age: an analysis of outcomes and complications. Muscle Nerve 2013; 48:770-76

6. Engh JA, Horowitz M, Burkhart L, et al. Repeat microvascular decompression for hemifacial spasm. J Neurol Neurosurg Psychiatry 2005;76:1574-80

7. Hughes MA, Branstetter BF, Frederickson AM, et al. Imaging hemifacial spasm. Neurographics 2015;5:2-8

8. Tomii M, Onoue H, Yasue M, et al. Microscopic measurement of the facial nerve root exit zone from central glial myelin to peripheral Schwann cell myelin. J Neurosurg 2003;99:121-24

9. Campos-Benitez M, Kaufmann AM. Neurovascular compression findings in hemifacial spasm. J Neurosurg 2008;109:416-20

10. Sekula RF Jr, Bhatia S, Frederickson AM, et al. Utility of intraoperative electromyography in microvascular decompression for hemifacial spasm: a meta-analysis. Neurosurg Focus 2009;27:E10

11. Wilson CB. Neurosurgical Procedures: Personal Approaches to Classic Operations. Baltimore: Lippincott Williams \& Wilkins; 1992

12. Linskey ME, Ratanatharathorn V, Penagaricano J. A prospective cohort study of microvascular decompression and gamma knife surgery in patients with trigeminal neuralgia. J Neurosurg 2008; 109(suppl):160-72

13. Zhong J, Li ST, Zhu J, et al. A clinical analysis on microvascular decompression surgery in a series of 3000 cases. Clin Neurol Neurosurg 2012;114:846-51

14. Barker FG 2nd, Jannetta PJ, Bissonette DJ, et al Microvascular decompression for hemifacial spasm. J Neurosurg 1995;82:201-10

15. McLaughlin MR, Jannetta PJ, Clyde et al. Microvascular decompression of cranial nerves: lessons learned after 4400 operations. J Neurosurg 1999;90:1-8 
16. Wang X, Thirumala PD, Shah A, et al. Microvascular decompression for hemifacial spasm: focus on late reoperation. Neurosurg Rev 2013; 36:637-43; discussion 643-44

17. Sheth S, Branstetter BF 4th, Escott EJ. Appearance of normal cranial nerves on steady-state free precession MR images. Radiographics 2009;29:1045-55

18. Raslan AM, DeJesus R, Berk C, et al. Sensitivity of high-resolution three-dimensional magnetic resonance angiography and three-dimensional spoiled-gradient recalled imaging in the prediction of neurovascular compression in patients with hemifacial spasm. J Neurosurg 2009;111:733-36
19. Iijima K, Horiguchi K, Yoshimoto Y. Microvascular decompression of the root emerging zone for hemifacial spasm: evaluation by fusion magnetic resonance imaging and technical considerations. Acta Neurochir (Wien) 2013;155:855-62

20. Kanoto M, Toyoguchi Y, Hosoya T, et al. Visualization of the trochlear nerve in the cistern with use of high-resolution turbo spin-echo multisection motion-sensitized driven equilibrium. AJNR Am J Neuroradiol 2013;34:1434-37

21. Sekula RF Jr, Frederickson AM, Branstetter BF, et al. Thin-slice T2 MRI imaging predicts vascular pathology in hemifacial spasm: a case-control study. Mov Disord 2014;29:1299-303 